\title{
Investigating the relationship between foods consumed and eating location for UK adolescents using the NDNS
}

\author{
A.N. Chapman ${ }^{1}$, E. Almiron-Roig ${ }^{2}$, G.K. Pot ${ }^{3}$ and L. Palla ${ }^{1}$ \\ ${ }^{1}$ Dept Medical Statistics, LSHTM, London, \\ ${ }^{2} M R C H N R$ Cambridge and \\ ${ }^{3}$ Diabetes \& Nutritional Sciences Division, Faculty of Life Sciences \& Medicine, King's College London
}

The increasing rate in obesity amongst young people is a national concern. Recently the location of eating has been implicated in changes in diet quality in adolescents ${ }^{(1)}$. Data from four day food diaries from 884 teenagers aged 11-18 years participating in the 2008-2012 UK National Dietary and Nutrition Survey Rolling Programme (NDNS RP) were used to investigate the relationship between location and food group consumption by teenagers. Analysis focussed on the 25 major food groups which contribute $80 \%$ of teenagers' calories.

Confidence regions as "ellipses" on Correspondence Analysis (CA) plots were used to identify five food-location combinations significantly distinct from average in the randomly selected 20,567 diary records used as the hypothesis generating sample.

Logistic regression was used to model the data by Generalised Estimating Equations in the remaining 20,455 food diary records representing the hypothesis testing sample. Adjusted odds-ratios (ORs) were estimated for the population average effect of location on consumption for five food groups emerged via CA. The table of results gives adjusted ORs for food groups at "Other" locations compared to Home, and also to School/Work locations. "Other" locations were defined as: Leisure locations such as sports clubs, cafes and fast-food, Mobile locations such as a bus or a car, Friends' and Carers' homes, and Miscellaneous locations such as the "High street". Odds ratios were estimated with adjustment for the effect of gender, age, socio-economic classification and weekend/weekday. The significance level was set at $1 \%$.

\begin{tabular}{lccc}
\hline Food Group & OR vs Home & $99 \%$ CI $\mathrm{p}$-value & OR vs School/Work \\
\hline 1. Sweetened soft drinks & $\mathbf{2 \cdot 9}$ & $(2 \cdot 3,3 \cdot 5) \mathrm{p}<0 \cdot 0001$ & $\mathbf{2 \cdot 3}$ \\
2. Chips & $\mathbf{2 \cdot 8}$ & $(2 \cdot 2,3 \cdot 6) \mathrm{p}<0 \cdot 0001$ & $\mathbf{3} \cdot \mathbf{4}$ \\
3. Chocolate & $\mathbf{2 \cdot 5}$ & $(1 \cdot 8,3 \cdot 4) \mathrm{p}<0 \cdot 0001$ & $\mathbf{1 \cdot 8}$ \\
4. Meat pastries & $\mathbf{2 \cdot 8}$ & $(1 \cdot 5,5 \cdot 0) \mathrm{p}<0 \cdot 0001$ & $1 \cdot 3 \cdot 1) \mathrm{p}<0 \cdot 0001$ \\
5. Fruit & $\mathbf{0 . 7 0}$ & $(0 \cdot 49,0 \cdot 98) \mathrm{p}=0 \cdot 006$ & $(1 \cdot 2,2 \cdot 8) \mathrm{p}=0.0002$ \\
\hline
\end{tabular}

These ORs estimate that, for example, when a teenager finds himself/herself at a location "Other" then the odds are 2.9 times higher than at Home that they will consume a sweetened soft drink, and 2.3 times higher than at School or Work. Additionally, we found that teenagers from families in a "lower" socio-economic category eat chips and soft-drinks more often, but fruit less often. Older teenagers eat meat pies more often, but fruit less often. These results suggest that teenagers are not making healthy food choices when choosing food at "Other" locations. "Other" locations are often commercial locations, and it may be important to develop incentives, perhaps through legislation, for food businesses to promote healthy choices and penalise less-healthy choices.

1. Adams et al. (2015) International Journal of Behavioural Nutrition and Physical Activity 12:51. 